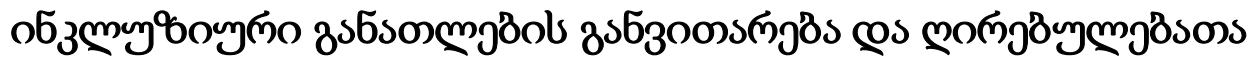

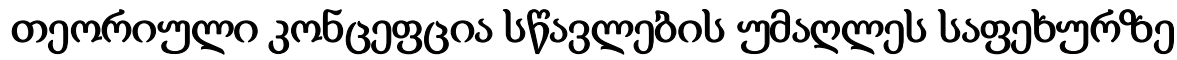

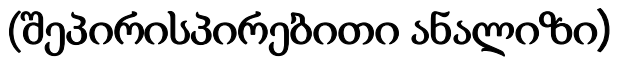

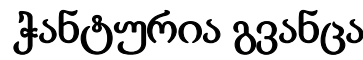

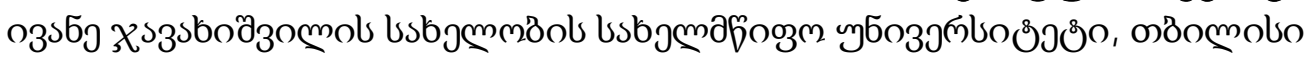

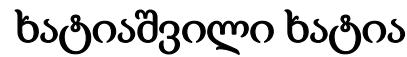

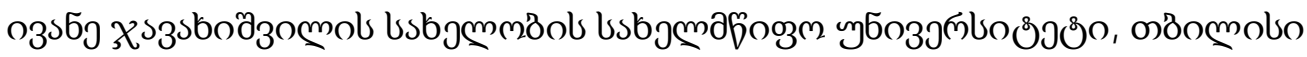

https://doi.org/10.52340/idw .2021.546

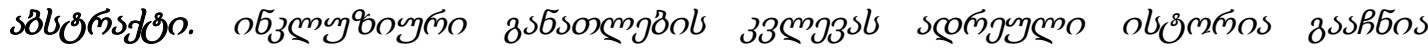

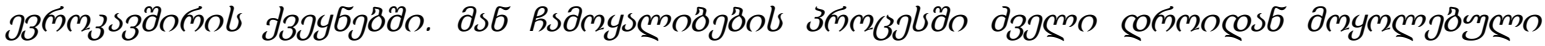

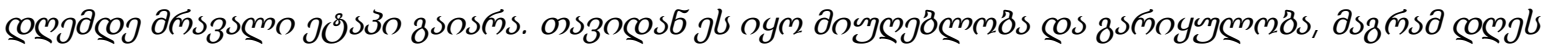

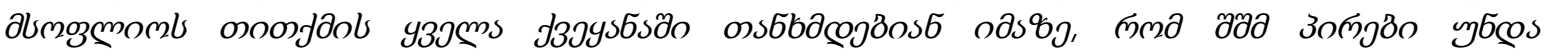

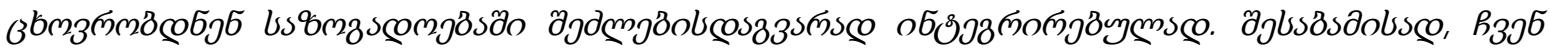

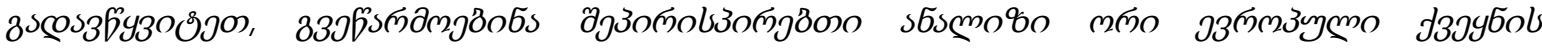
aszsलmoos 8 .

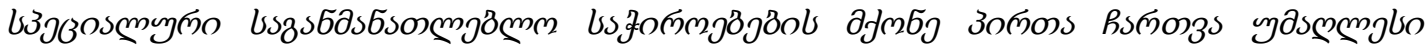

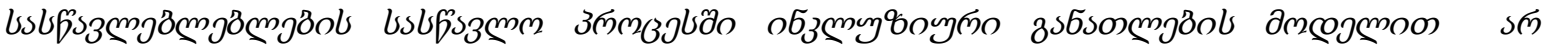

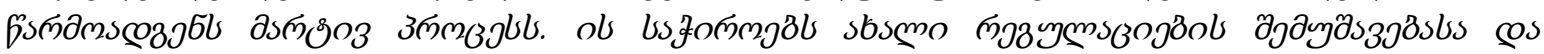

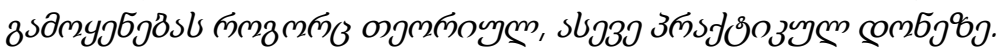

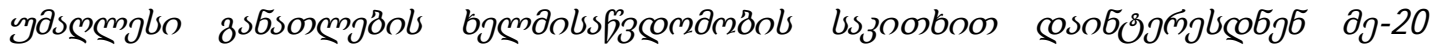

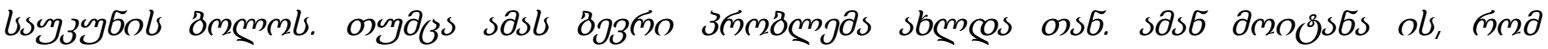

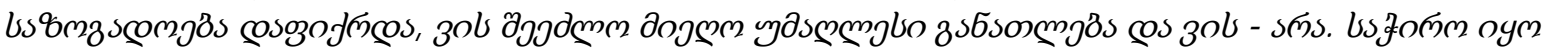

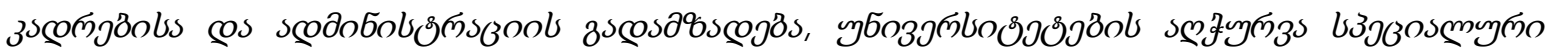

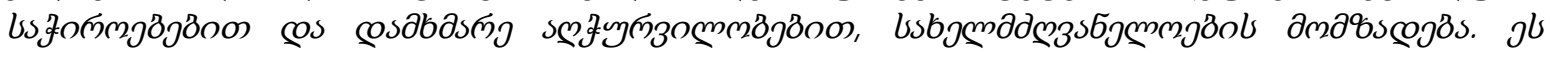

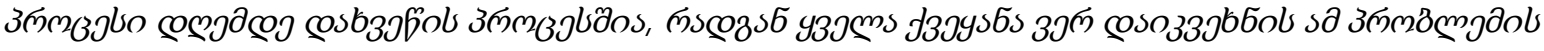

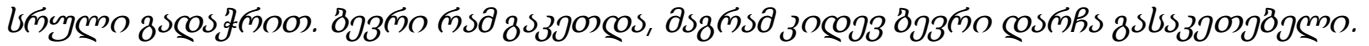

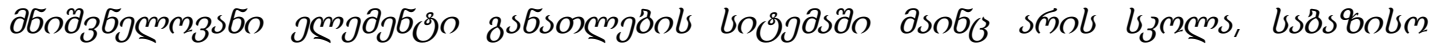

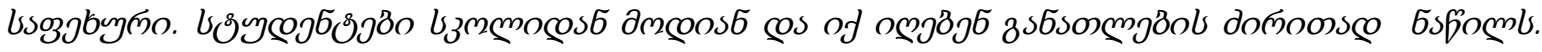

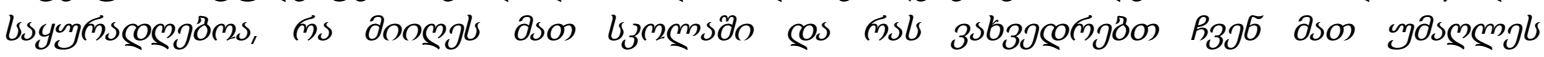
uslfis

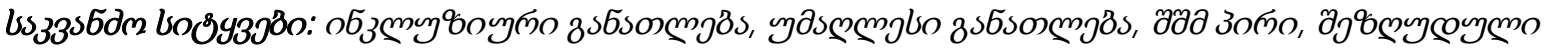

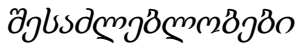

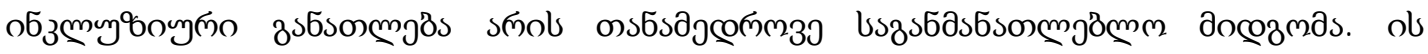

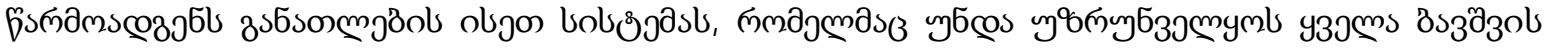

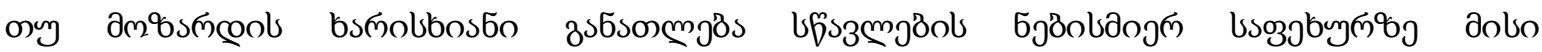

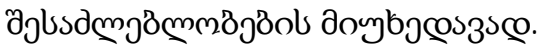

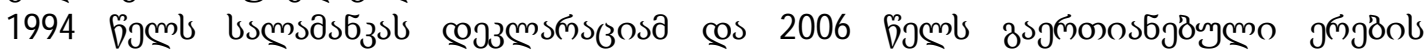

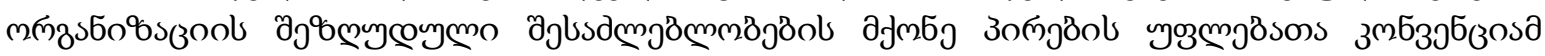

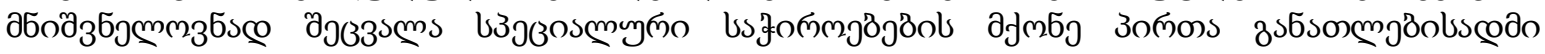

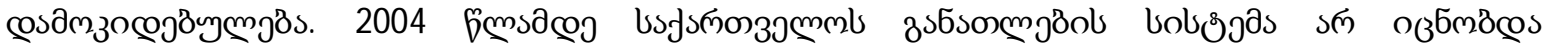




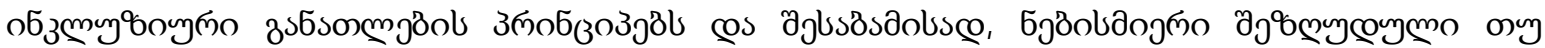

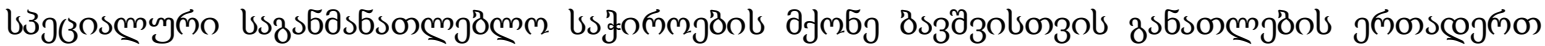

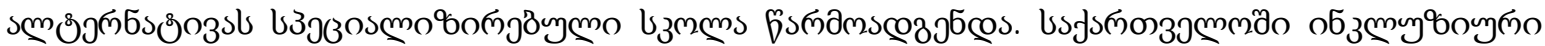

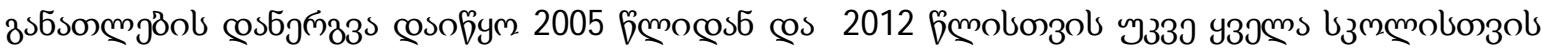
uszsmejörymm zstbes.

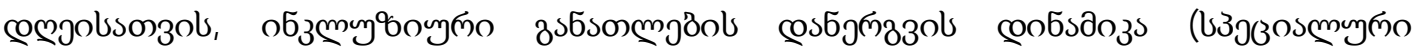

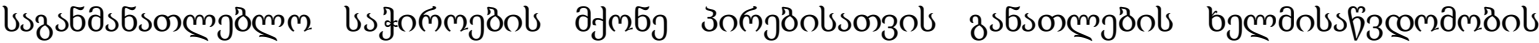

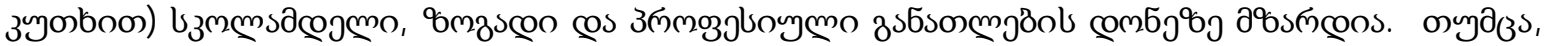

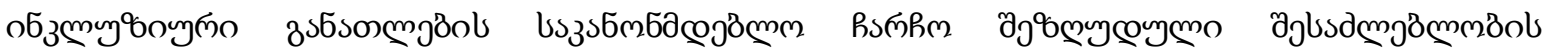

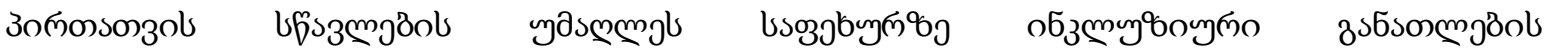

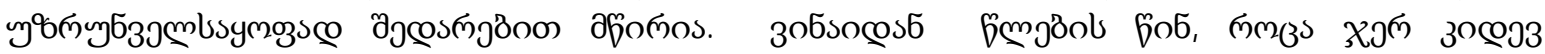

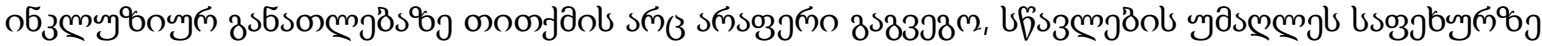

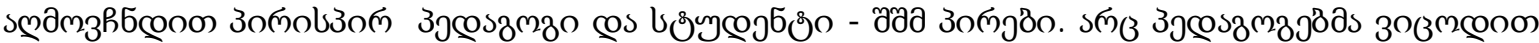

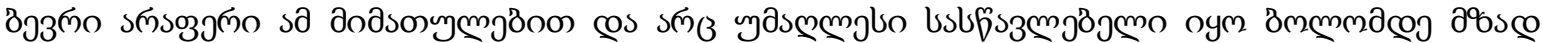

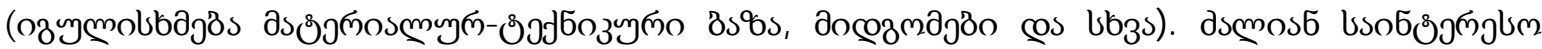

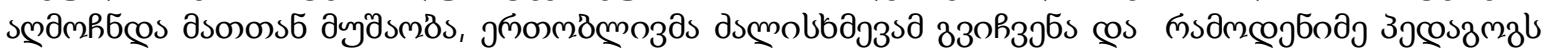

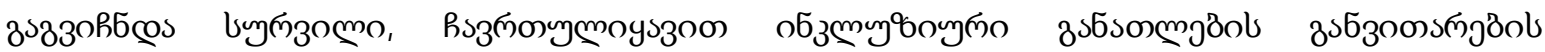

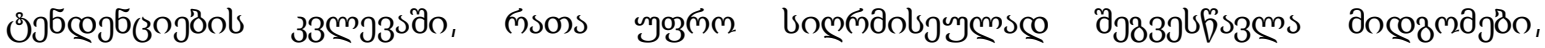

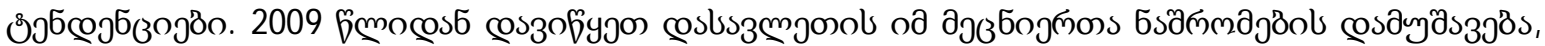

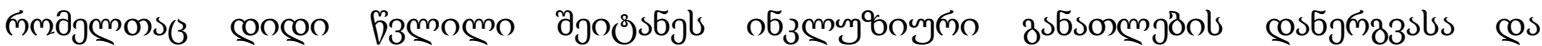

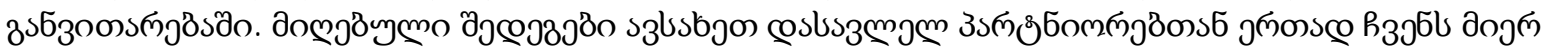

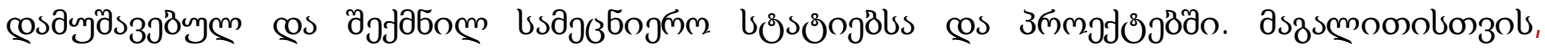

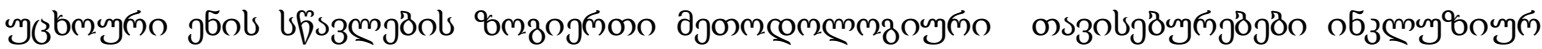

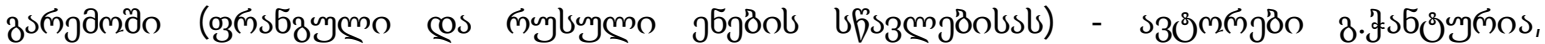
b.bsososðzomo, Психо-социальные сложности в изучении французского и русского языков в

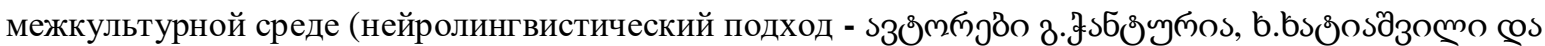

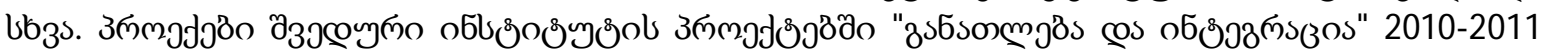

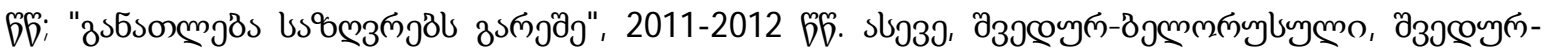

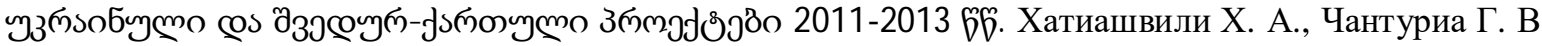
Обучение инностранному языку в инклюзивной среде (Опыт Грузии), Япония, Киото, 2019г.

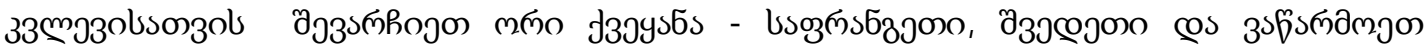

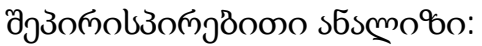

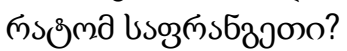

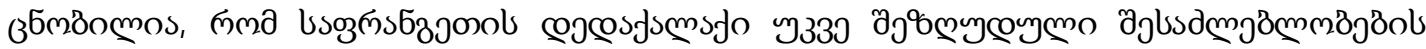

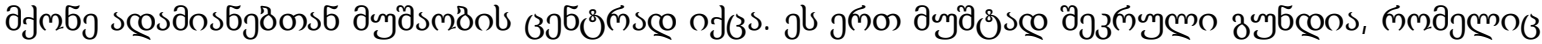

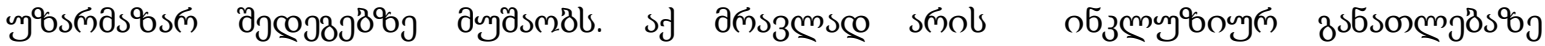

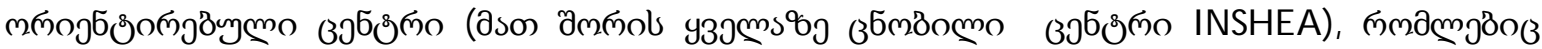

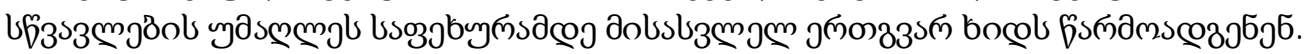

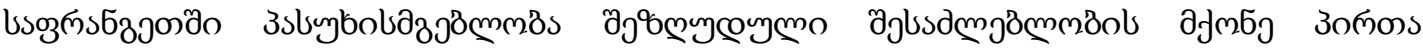

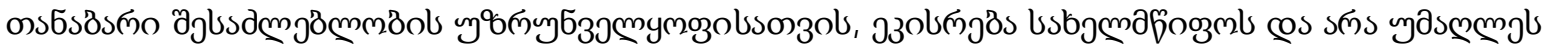

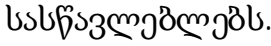

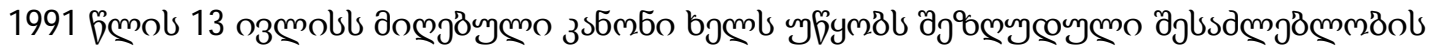

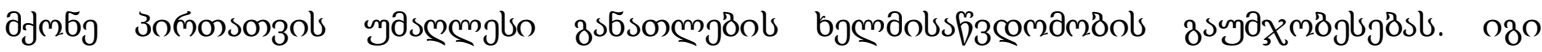

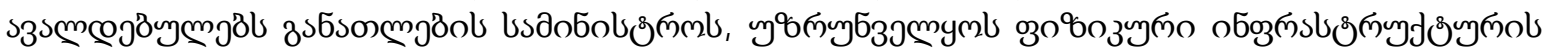

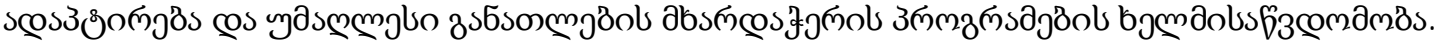

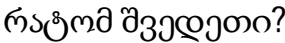

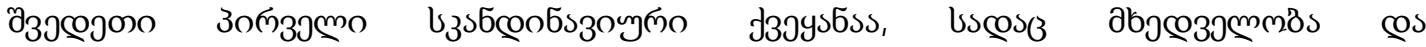

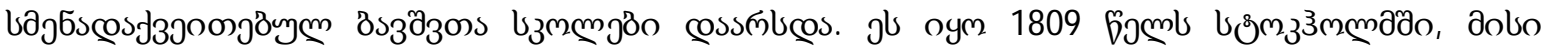

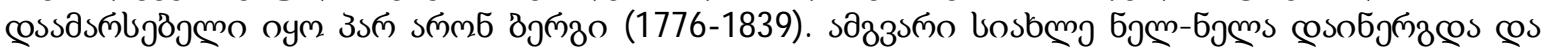

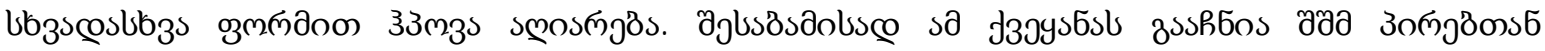




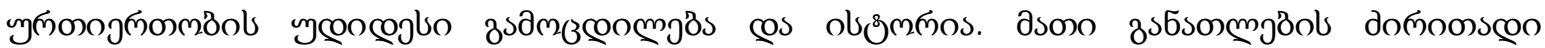

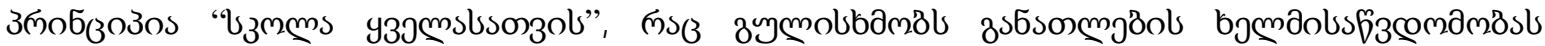

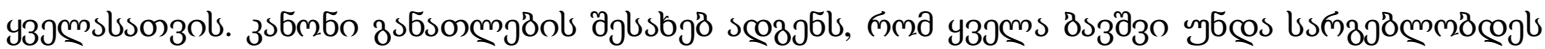

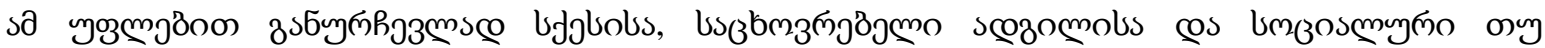

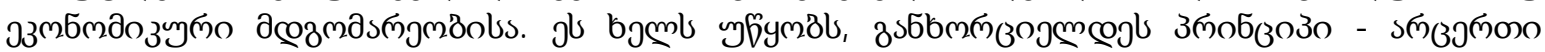

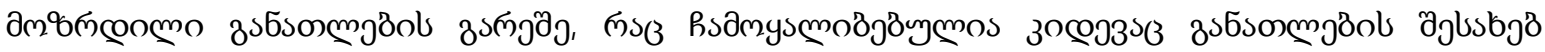

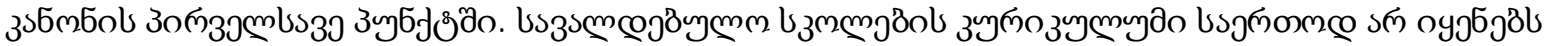

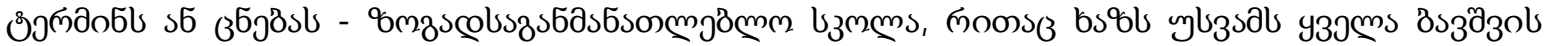

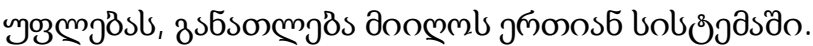

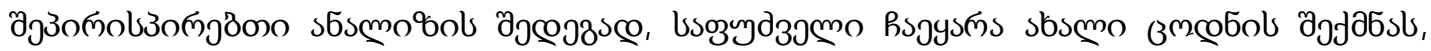

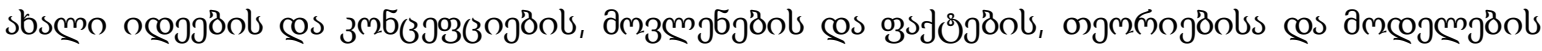

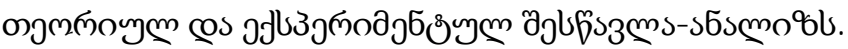

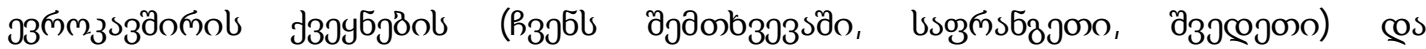

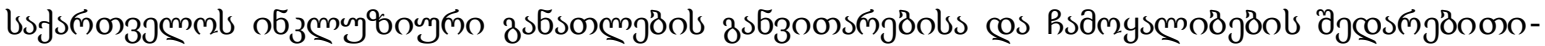

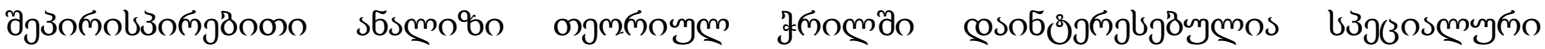

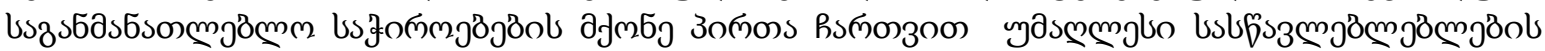

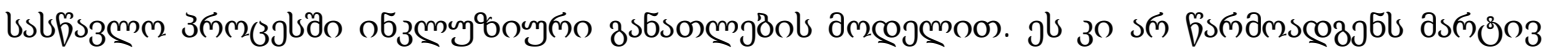

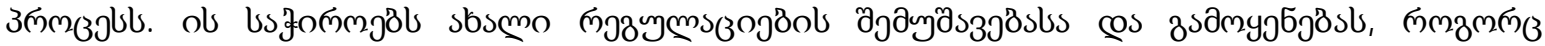

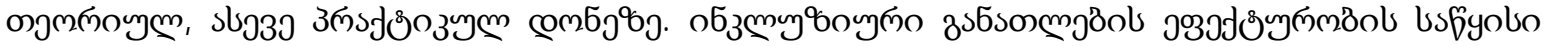

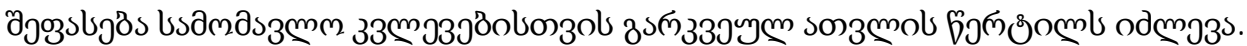

bso6о̊

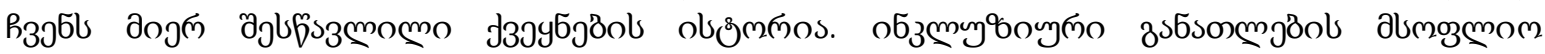

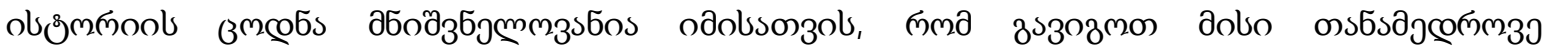

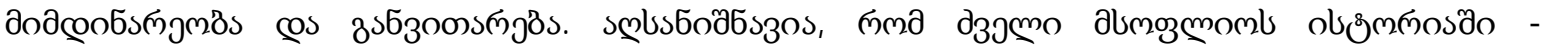

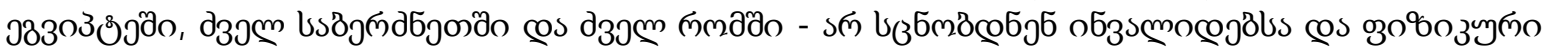

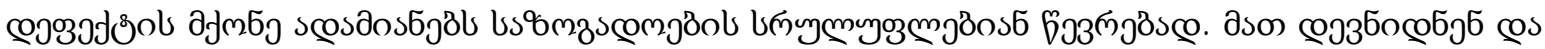

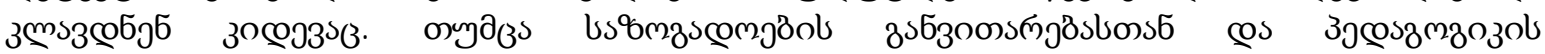

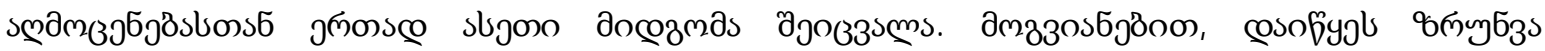

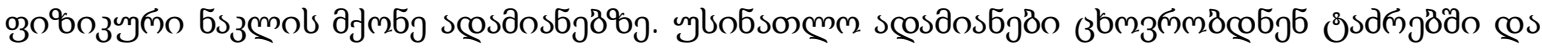

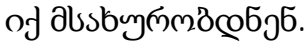

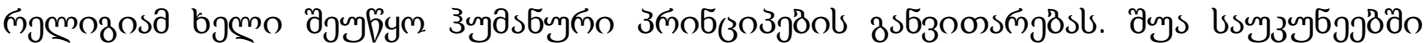

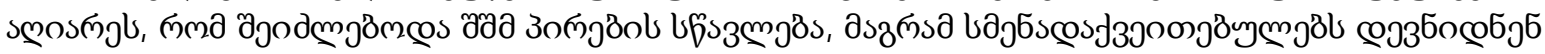

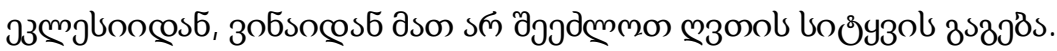

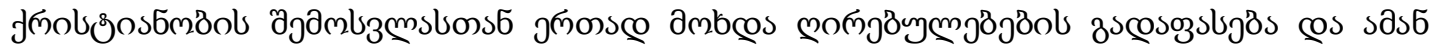

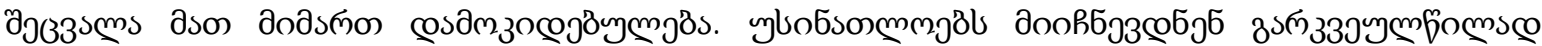

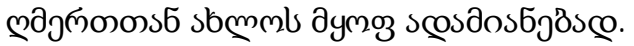

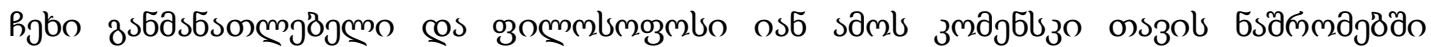

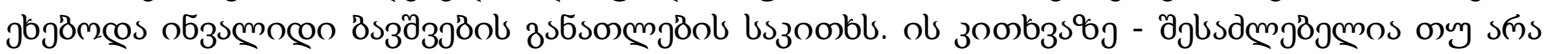

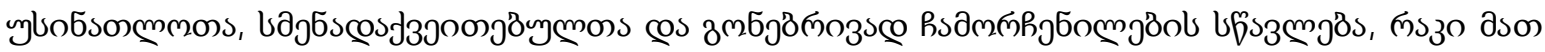

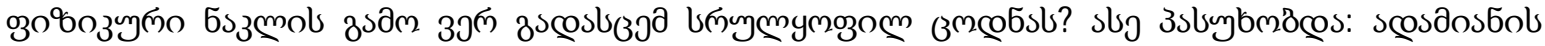

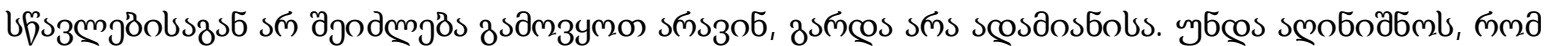

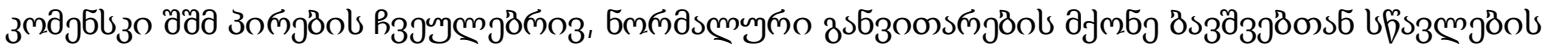

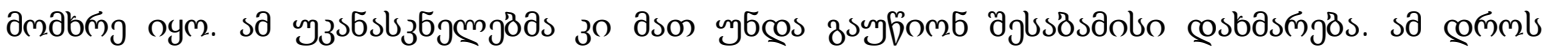

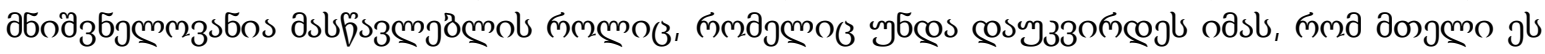

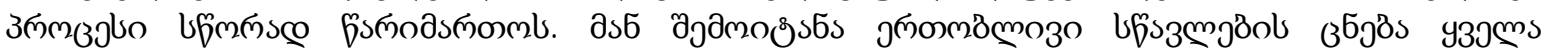

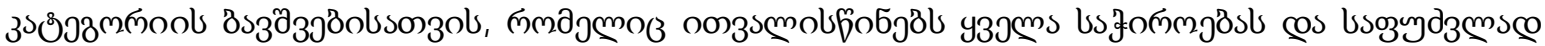

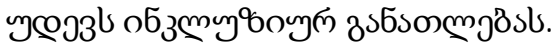

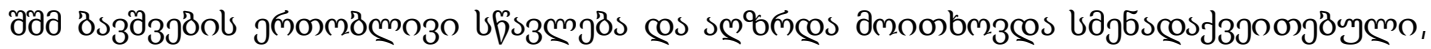

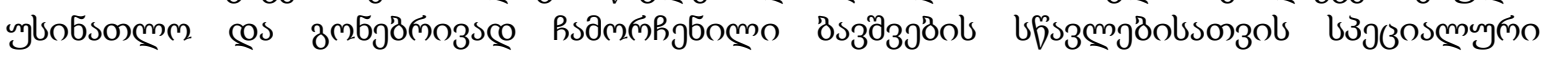

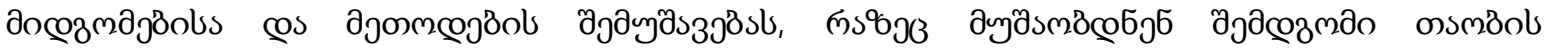




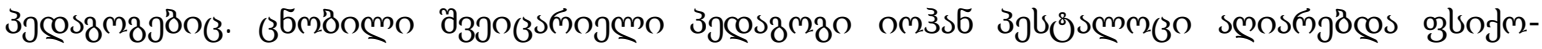

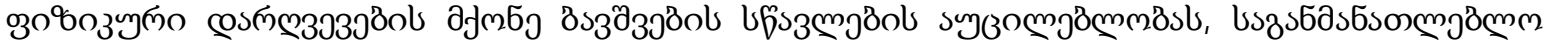

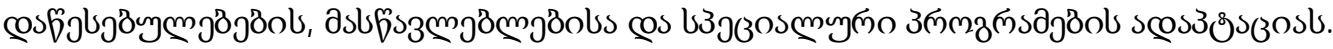

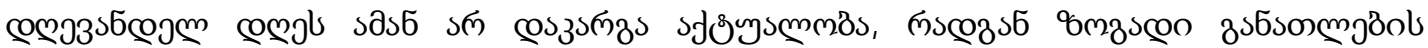

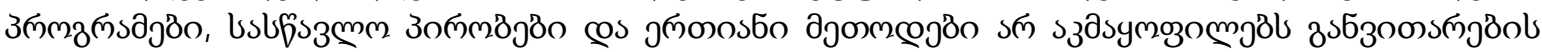

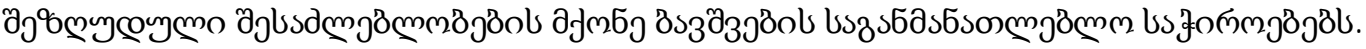

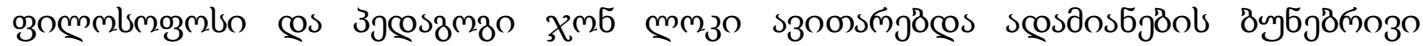

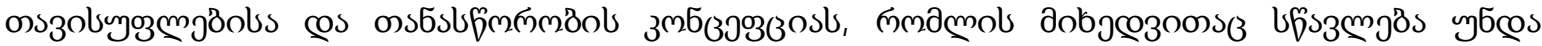

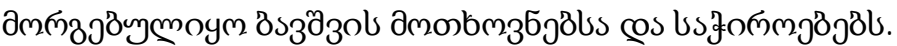

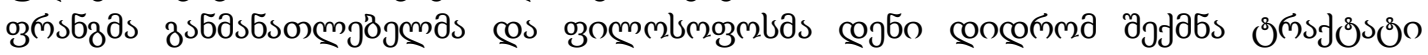

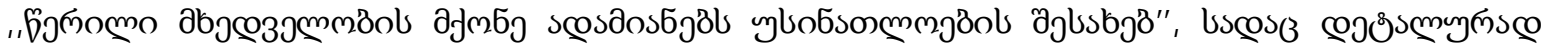

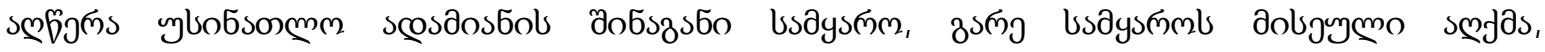

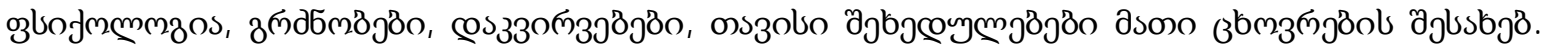

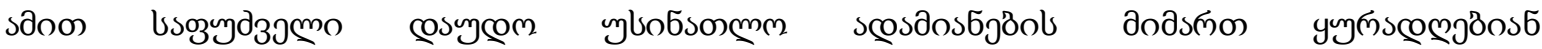

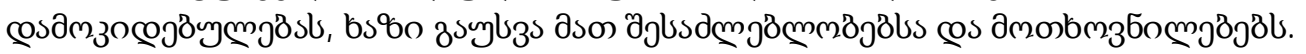

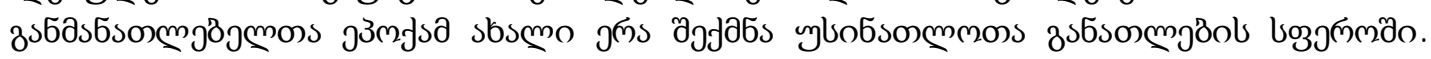

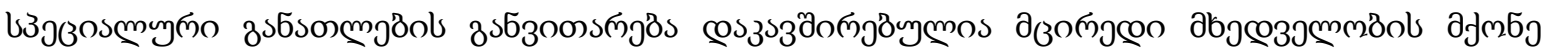

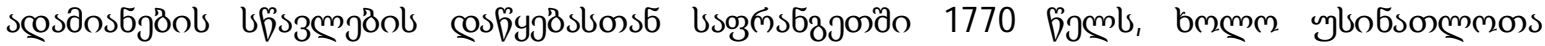

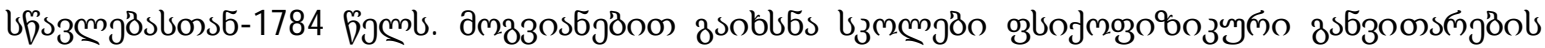

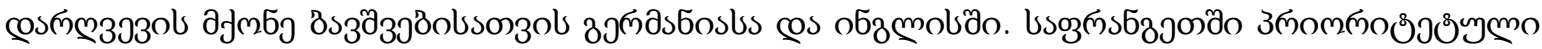

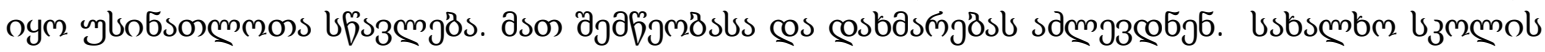

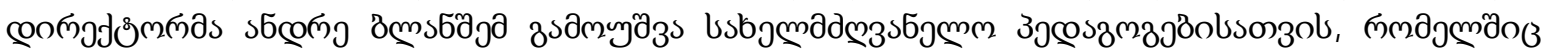

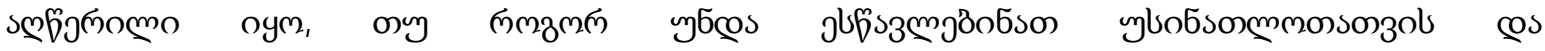

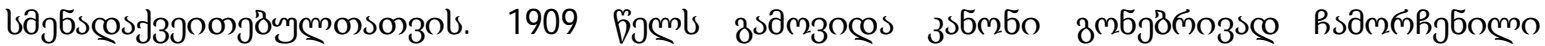

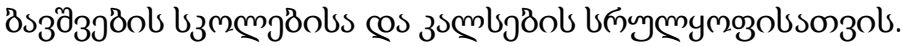

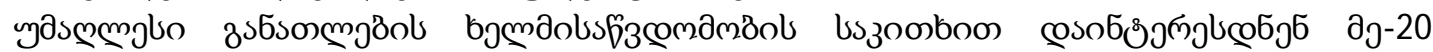

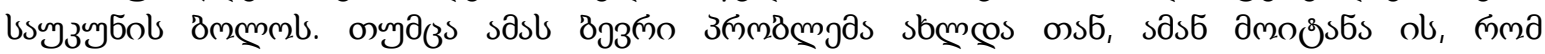

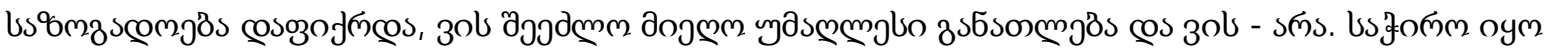

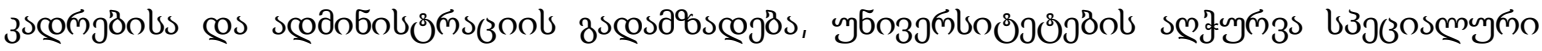

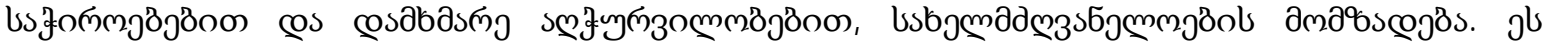

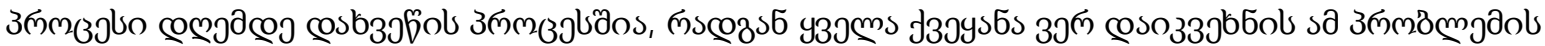

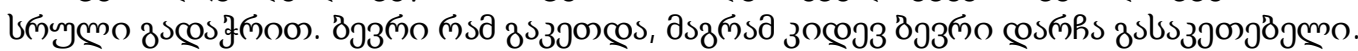

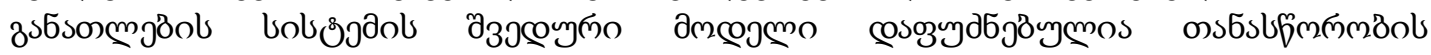

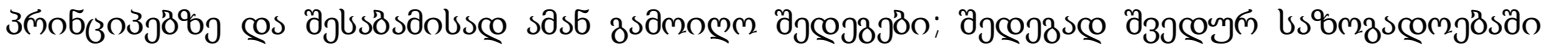

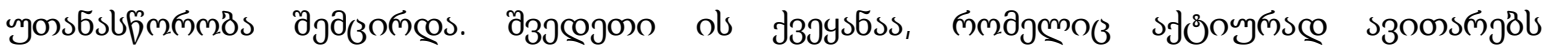

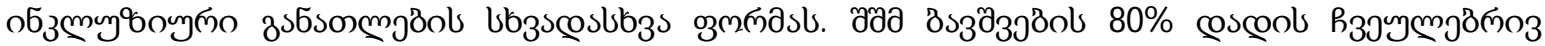

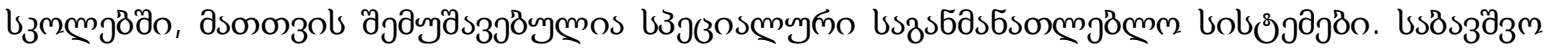

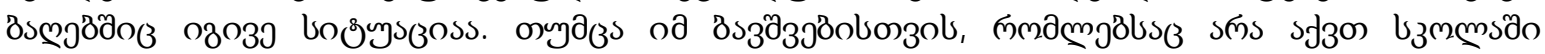

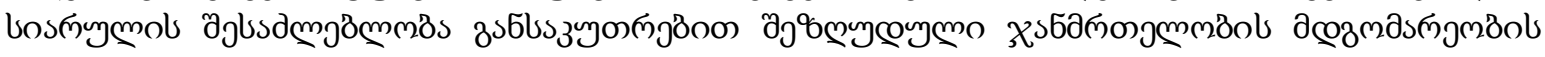

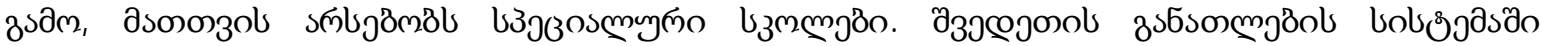

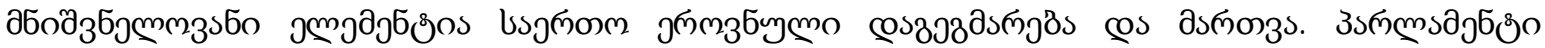

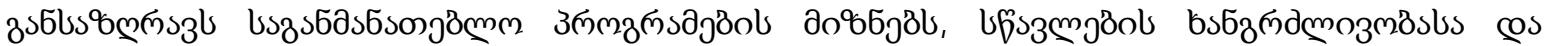

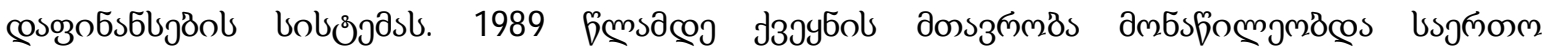

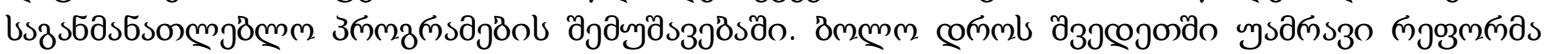

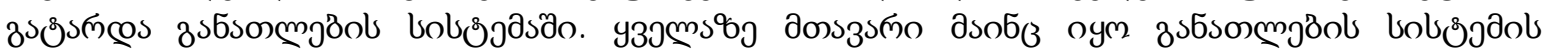

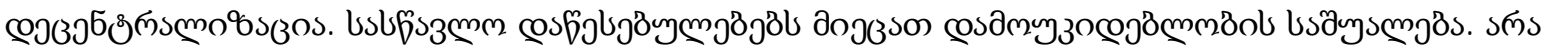

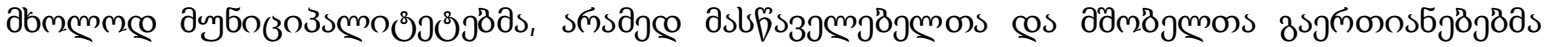

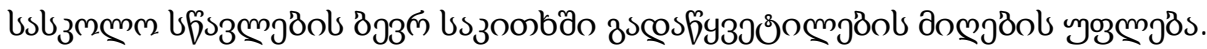

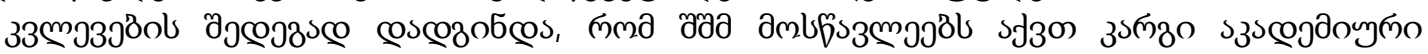

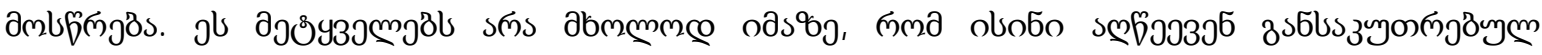




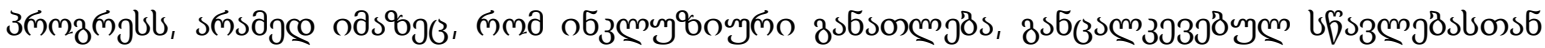

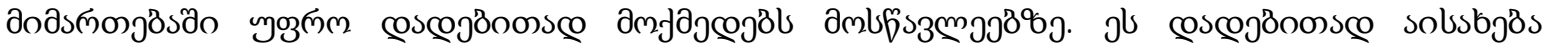

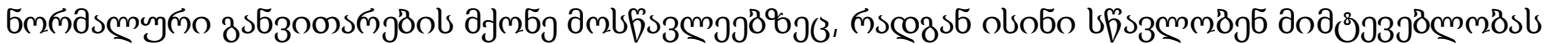

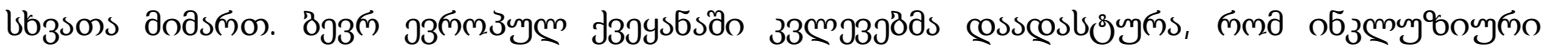
дsБsоm

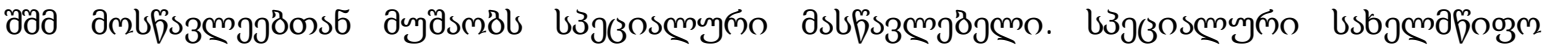

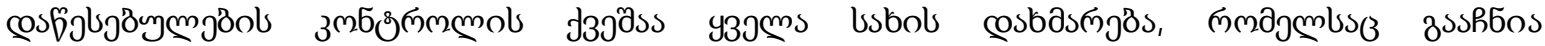

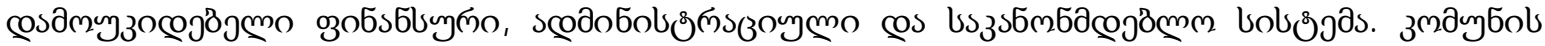

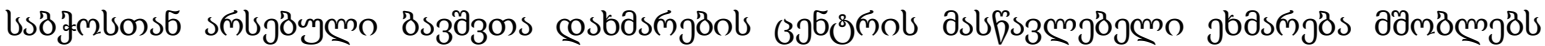

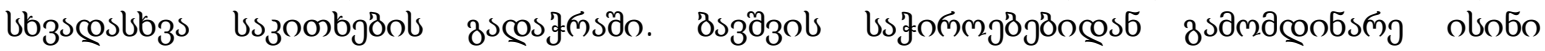

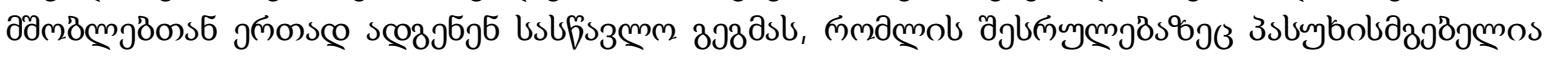

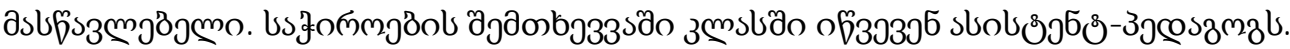

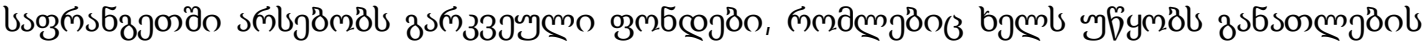

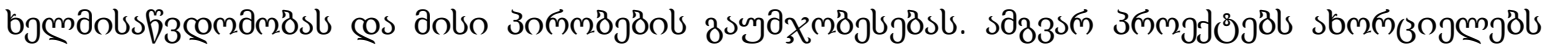

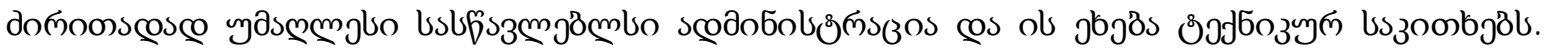

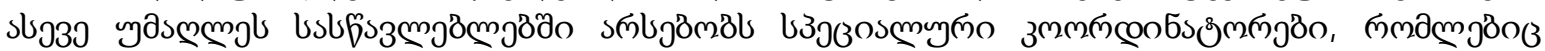

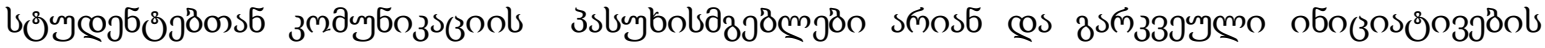

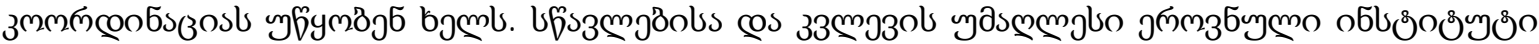

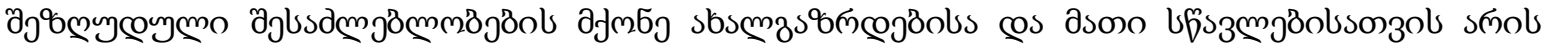

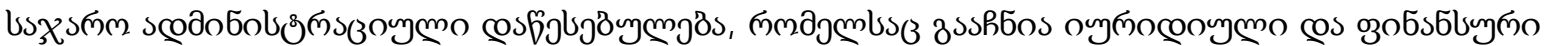

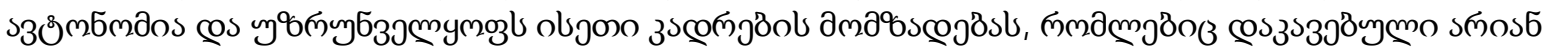

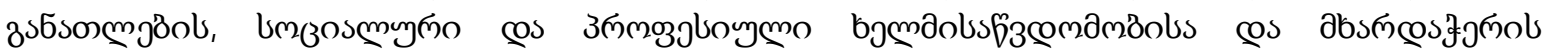
uszoobjönom.

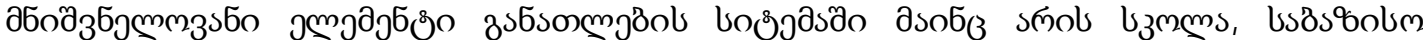

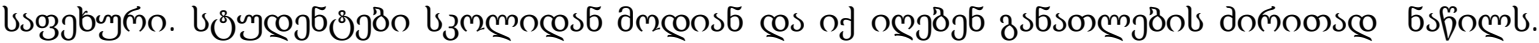

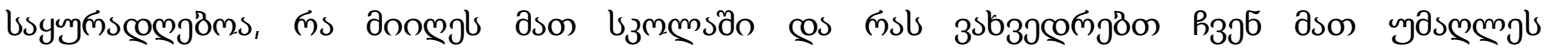

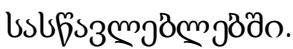

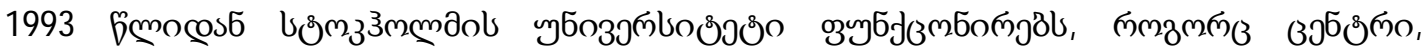

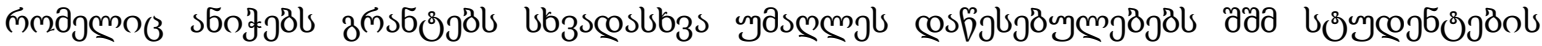

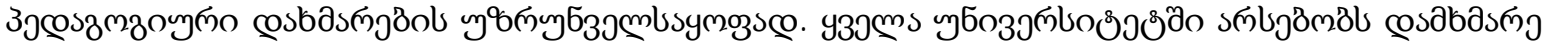

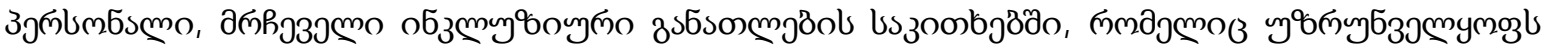

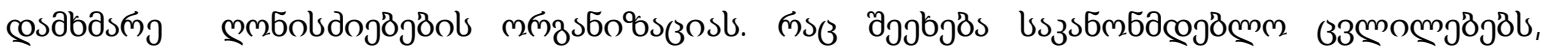

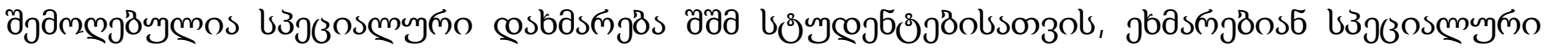

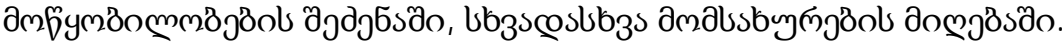

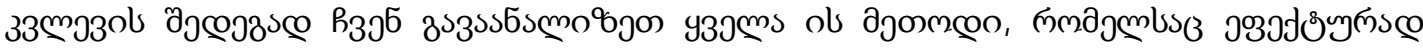

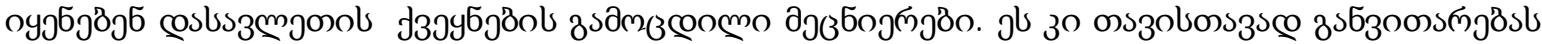

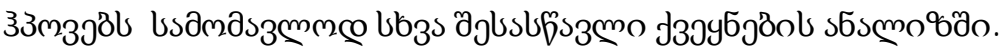

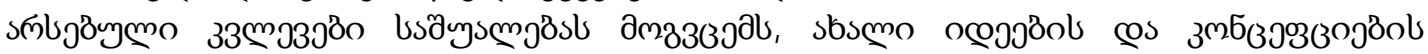

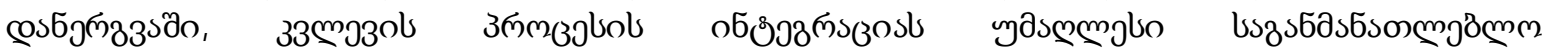

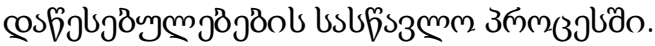

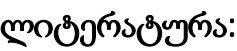

1. Mirza, H. S. Race, gender and educational desire Text. / H. S. Mirza Equality, participation and inclusion 1: Diverse perspectives (2nd ed.);in Rix J., et. al. (Eds.). London and New York: Routledge and O pen University Press, 2010.

2. Hoover, J. J. The role of special educators in a multitiered instructional system Text. / J. J.Hoover, J. R.Patton // Intervention jn JSchjDol .and-Clinic.2008.-№ 43

3. Gardner, H. Intelligence reframed: Multiple intelligences for the 21st century Text. / H. Gardner. New Y ork: Basic Books, 2000

4. Малофеев, Н. Н. Интеграция и специальные образовательные учреждения Текст. / 
5. Малофеев Н. Н., Н. Д. Шматко // Дефектология. 2008

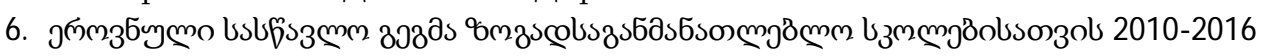

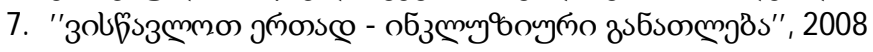

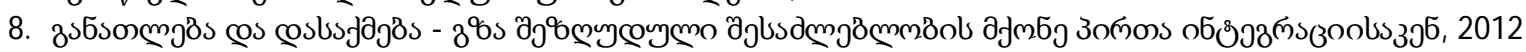

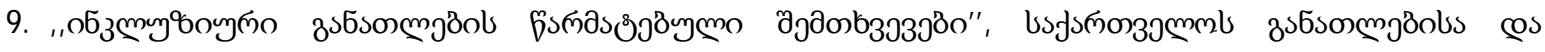

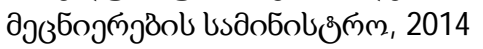

\title{
Development of Inclusive Education and Theoretical Concept of Values in the Higher Education System (cross-case analysis)
}

\author{
Chanturia Gvanca \\ Ivane Javaxishvili State University, Tbilisi \\ Khatiashvili Khatia \\ I vane Javaxishvili State University, Tbilisi
}

\begin{abstract}
The study of inclusive education has an early history in EU countries. It has gone through many stages in its formation from ancient times to the present day. At first the persons with disabilities were excluded from society, but today almost every country in the world agrees, that people with disabilities should live integrated in our society as it is possible. Therefore, we decided to conduct a cross-case analysis on the example of two European countries - France and Sweden.

Involving persons with special educational needs in the educational process of HEI on the example of inclusive education model is not an easy process. It requires the development and application of new regulations at both, theoretical and practical levels.

Authorities were interested in the problem of access to the HEI primarily in the late $20^{\text {th }}$ century. However, this was accompanied with many problems. This led the public to think about who could get a higher education and who could not. It was necessary to train staff and administration, to equip universities with special equipment and prepare textbooks. This is still in the process of refinement, as not all countries can boast of a complete solution to this problem. Much has been done, but much more remains to be done.

An important element in the education system is still the school, the basic level. Students come from school and get the bulk of their education there. It is noteworthy, what they got from school and what we could give them in the high school.
\end{abstract}

Key-words: Inclusive education, Higher education, Persons with special educational needs, Disabilities 